\title{
Emergence of energy dependence in the fragmentation of heterogeneous materials
}

\author{
Gergő Pál, ${ }^{1}$ Imre Varga, ${ }^{2}$ and Ferenc Kun ${ }^{1, *}$ \\ ${ }^{1}$ Department of Theoretical Physics, University of Debrecen, P.O. Box 5, H-4010 Debrecen, Hungary \\ ${ }^{2}$ Department of Informatics Systems and Networks, University of Debrecen, P.O. Box 12, H-4010 Debrecen, Hungary
}

(Received 26 August 2014; published 19 December 2014)

\begin{abstract}
The most important characteristics of the fragmentation of heterogeneous solids is that the mass (size) distribution of pieces is described by a power law functional form. The exponent of the distribution displays a high degree of universality depending mainly on the dimensionality and on the brittle-ductile mechanical response of the system. Recently, experiments and computer simulations have reported an energy dependence of the exponent increasing with the imparted energy. These novel findings question the phase transition picture of fragmentation phenomena, and have also practical importance for industrial applications. Based on large scale computer simulations here we uncover a robust mechanism which leads to the emergence of energy dependence in fragmentation processes resolving controversial issues on the problem: studying the impact induced breakup of platelike objects with varying thickness in three dimensions we show that energy dependence occurs when a lower dimensional fragmenting object is embedded into a higher dimensional space. The reason is an underlying transition between two distinct fragmentation mechanisms controlled by the impact velocity at low plate thicknesses, while it is hindered for three-dimensional bulk systems. The mass distributions of the subsets of fragments dominated by the two cracking mechanisms proved to have an astonishing robustness at all plate thicknesses, which implies that the nonuniversality of the complete mass distribution is the consequence of blending the contributions of universal partial processes.
\end{abstract}

DOI: 10.1103/PhysRevE.90.062811

PACS number(s): 89.75.Da, 46.50.+a, 05.10.-a

\section{INTRODUCTION}

Fragmentation into numerous pieces occurs when a large amount of energy is imparted to a solid within a short time [1]. Impact induced fragmentation of heterogeneous materials is abundant in nature having also a high importance for industrial applications especially in mining and ore processing [2-8]. During the past decades research on fragmentation mainly focused on the statistics of fragment masses (sizes) obtained by the breakup of heterogeneous materials $[1,9,10]$. A large number of experimental [1,5-17] and theoretical studies [13,18-24] have confirmed that the mass distribution of fragments is described by a power law functional form. The exponent of the distribution was found to show a high degree of robustness, i.e., investigations revealed that the value of the exponent does not depend on the type of materials, amount of input energy, and on the way the energy is imparted to the system until materials of a high degree of heterogeneity are fragmented $[1,9,10]$. The value of the exponent is mainly determined by the dimensionality of the system $[13,15,18,19,21,23,25-27]$ and by the brittle or ductile mechanical response of the material [28]. The universality of fragmenting has been shown to be the fingerprint of an underlying phase transition from the damaged to the fragmented phase of the breakup process [5,6,14,18].

Recently, experiments on the impact induced fragmentation of long thin glass rods [29,30] and freely hanging glass plates [31,32] revealed energy dependence of the mass distribution exponent, i.e., the exponent was found to increase with the imparted energy [29-35]. The importance of these findings originates from the fact that, on the one hand, they question the universality and hence the phase transition interpretation

\footnotetext{
*ferenc.kun@science.unideb.hu
}

of fragmentation phenomena, and on the other hand, they have consequences on the design of engineering technologies used for crushing in mining and ore processing [10]. Recent computer simulations have also provided an interesting counterexample [23]: mass distributions of pieces obtained by the breakup of spherical bodies impacting against a hard wall have been found to get steeper with increasing impact velocity. However, it proved to be an apparent energy dependence which occurs solely due to the moving cutoff of the mass distributions, and hence, it can be transformed out by rescaling with the average fragment mass [23]. This study highlighted the importance of scaling and data collapse analysis when evaluating fragmentation results of finite size systems.

In order to resolve controversial issues on the energy dependence of the exponent of fragment mass distributions, in the present paper we study the impact induced breakup of heterogeneous materials by large scale computer simulations. Our results demonstrate that energy dependence emerges when the fragmenting object is embedded in a higher dimensional space. Studying the fragmentation of platelike objects in three dimensions we show that energy dependence is obtained for low plate thicknesses, while it disappears for thick plates. The reason is that due to the interplay of the geometry of the sample and of the embedding space a transition takes place in the system between two fragmentation mechanisms as the impact velocity is increased: At low velocities the crack structure is determined by the interference of elastic waves resulting in an essentially two-dimensional crack pattern with a regular structure. High velocity impact gradually excites cracking in the $3 \mathrm{D}$ bulk of the solid giving rise to a highly disordered crack structure and a steeper decay of the mass distribution. In three-dimensional (3D) bulk samples the transition is hindered so that a unique exponent emerges. In spite of the observed nonuniversality of the complete mass distribution, identifying subsets of fragments dominated by different cracking 
mechanisms an astonishing universality of their mass distributions is revealed at all plate thicknesses. Our study provides a robust scenario which leads to the energy dependence of mass distribution exponents in fragmentation phenomena but it still underlines the importance of universality.

\section{DISCRETE ELEMENT MODEL OF FRAGMENTATION}

We investigate the fragmentation of platelike objects induced by impact of a projectile in the framework of a discrete element model (DEM) developed recently [36]. The model has proven successful in reproducing key features of fracture processes of heterogeneous materials. Here we briefly summarize the main steps of the model construction based on Ref. [36]. Similar modeling approaches have been also used in Refs. [21,23,37].

The sample is represented as a random packing of spherical particles which was generated by sedimenting particles in a rectangular container [36,37]. The diameter $d$ of the particles was sampled from a uniform distribution in a narrow range $\langle d\rangle-\Delta d / 2 \leqslant d \leqslant\langle d\rangle+\Delta d / 2$, where $\langle d\rangle$ denotes the average diameter. The range $\Delta d$ of diameter values was set as $\Delta d /\langle d\rangle=0.05$. In the simulations platelike samples were constructed with a rectangular basis of side length $L$ and height $H$. Simulations were carried out with a fixed extension $L=30$ varying the height of the sample $H$ in the range $H=3-15$ measured in units of the average particle diameter $\langle d\rangle$. The total number of particles in the samples falls between 5000 $(H /\langle d\rangle=3)$ and $25000(H /\langle d\rangle=15)$.

In the model, cohesive interaction of particles is provided by beams which connect the particles along the edges of a Delaunay triangulation of the initial particle positions. In three dimensions the total deformation of a beam is calculated as the superposition of elongation, torsion, as well as bending and shearing. Crack formation is captured such that the beams, modeling cohesive forces between grains, can be broken according to a physical breaking rule, which takes into account the stretching and bending of contacts,

$$
\left(\frac{\varepsilon_{i j}}{\varepsilon_{\mathrm{th}}}\right)^{2}+\frac{\max \left(\Theta_{i}, \Theta_{j}\right)}{\Theta_{\mathrm{th}}} \geqslant 1 .
$$

Here $\varepsilon_{i j}$ denotes the axial strain of the beam between particles $i$ and $j$, while $\Theta_{i}$, and $\Theta_{j}$ are the bending angles of the beam ends. The parameters $\varepsilon_{\text {th }}$ and $\Theta_{\text {th }}$ control the relative importance of the two breaking modes [21,23,25,36,37]. In the model there is only structural disorder present, i.e., the breaking thresholds are constant $\varepsilon_{\text {th }}=0.002$ and $\Theta_{\text {th }}=2^{\circ}$, however, the physical properties of beams such as length, cross section, and elastic moduli are determined by the random particle packing. At the broken beams along the surface of the spheres cracks are generated inside the solid and as a result of the successive beam breaking the solid falls apart. The interaction of those particles which are not connected by beams, e.g., because the beam has been broken, is described by the Hertz contact law [38]. The Hertz contact ensures that force can be transmitted through crack faces when they are pressed against each other. The fragments are defined as sets of discrete particles connected by the remaining intact beams. The time evolution of the fragmenting solid is obtained by solving the equations of motion of the individual particles $[38,39]$ until

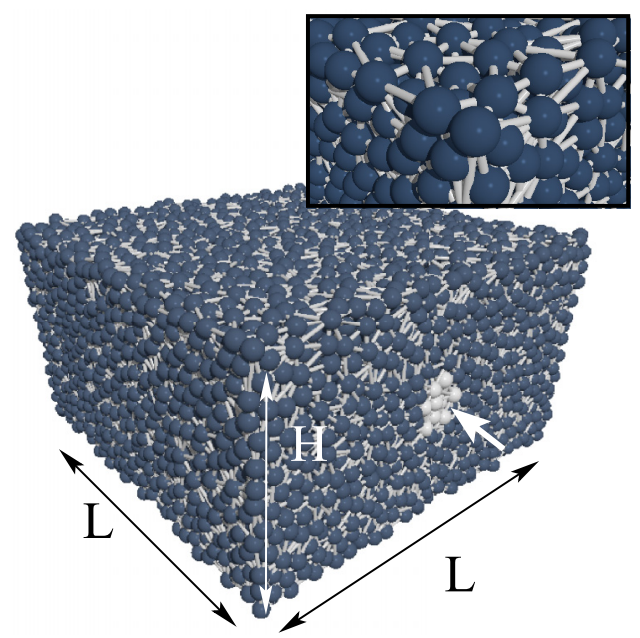

FIG. 1. (Color online) Geometrical setup of the simulations: rectangular samples of a square shaped basis were considered in such a way that the side length $L /\langle d\rangle=30$ of the square was fixed and the height $H /\langle d\rangle$ of the sample was varied from 3 to 15 . Here a sample is presented for $H /\langle d\rangle=11$. A particle in the middle of the front side of the sample was selected; together with its neighbors it got an initial velocity pointing into sample. The cylinders connecting the particles represent beams and the white arrow indicates the direction of the impact velocity. The inset shows a closer view on a small segment of the sample.

the entire system relaxes meaning that no beam breaking occurs during one thousand consecutive time steps and there is no energy stored in deformation. For more details of the model construction and parameter settings see Refs. [36].

Impact loading was performed in such a way that a single surface particle was selected in the middle of one of the side walls of the sample. Together with its contacting neighbors it got an initial velocity $\vec{v}_{0}$ pointing towards the center of mass of the body. This is equivalent to an experimental setup where the impactor does not penetrate the target but is stopped after hitting the target surface as, e.g., in Refs. [32,40]. The geometrical setup of the simulations and the loading condition of impact is illustrated in Fig. 1. For the smallest plate thickness $H /\langle d\rangle=3$ the impact site practically spans the cross section of the samples while for higher thicknesses the loading condition gets close to a pointlike impact.

Computer simulations were performed to determine the sound speed $c$ of the model material. In the presentation of the results lengths and velocities are made dimensionless by dividing them with the average particle diameter $\langle d\rangle$ and with the sound speed $c$, respectively.

\section{DAMAGE-FRAGMENTATION TRANSITION}

In order to investigate how the overall geometry of the system affects the outcomes of the breakup process for each plate thickness $H$ we carried out simulations varying the impact velocity in a broad range. To accumulate statistics simulations were repeated 2000 times for each parameter set with different realizations of the structural disorder. As a representative example Fig. 2 presents the time evolution of a plate of thickness $H /\langle d\rangle=3$ generated by an impact with 

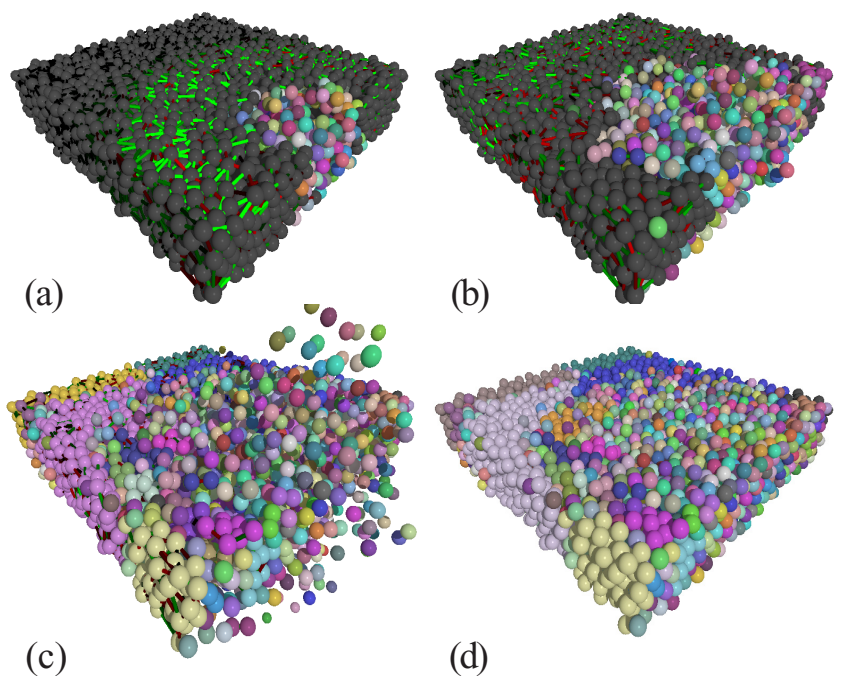

(d)

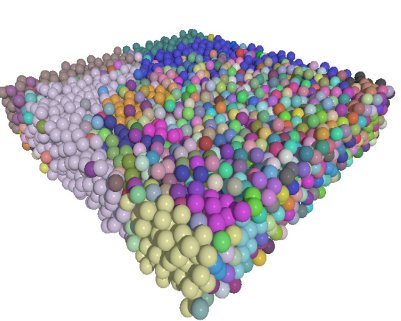

FIG. 2. (Color online) Time evolution of a fragmenting plate of thickness $H /\langle d\rangle=3$ embedded in the three-dimensional space. The impact velocity $v_{0} / c=0.2$ is slightly above the critical point of fragmentation. Compressed beams are green while the stretched ones are red so that the propagation of a shockwave can be observed. Particles are colored according to the fragment they belong to while the color (grey) of fragments is randomly selected from a palette. Panel (d) presents the final state of the system where the sample is reassembled by placing the particles back to their original position.

initial velocity $v_{0} / c=0.2$. The sample breaks into a large number of pieces due to the shockwave generated by the impact resulting in a broad distribution of fragment sizes. The figure also shows the final reassembled body where fragments can be easily identified. Of course, the degree of breakup strongly depends on the value of the impact velocity $v_{0}$ : at low $v_{0}$ the sample just gets damaged around the impact site, i.e., some beams break and small fragments comprising a few particles are ejected but the main part of the body retains its integrity. To achieve complete fragmentation, where even the largest fragment is significantly smaller than the original body, the impact velocity has to exceed a critical value $v_{c}$. To quantify the degree of breakup we determined the average mass of fragments $\left\langle M_{2} / M_{1}\right\rangle$ as the ratio of the second $M_{2}$ and first $M_{1}$ moments of fragment masses. The $k$ th moment $M_{k}$ of the fragment mass in a single simulation is defined as

$$
M_{k}=\sum_{i} m_{i}^{k}-m_{\max }^{k},
$$

where $m_{i}$ denotes the mass of single pieces, while $m_{\max }$ is the largest fragment mass. The sum runs over all fragments. The ratio of the two moments $M_{2} / M_{1}$ was determined in single simulations and then it was averaged over fragmentation events at each impact velocity $v_{0}$. The inset of Fig. 3 shows that gradually increasing $v_{0}$ the average fragment mass increases due to the creation of larger fragments. Since the largest fragment is always removed from the moments in Eq. (2), the decreasing branch of $\left\langle M_{2} / M_{1}\right\rangle$ is caused by the absence of a dominating piece. Hence, the position of the sharp maximum can be identified with the critical value $v_{c}$ of the impact velocity where complete breakup occurs. The result demonstrates that

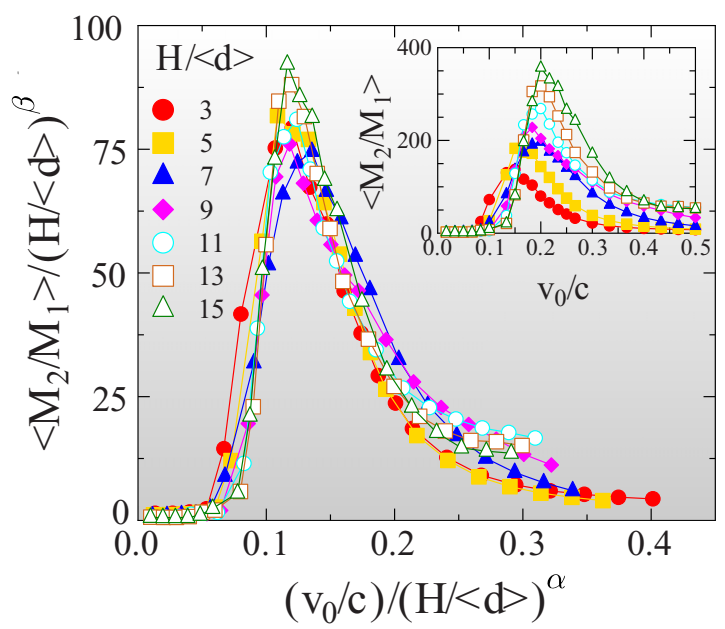

FIG. 3. (Color online) Inset: Average mass of fragments $\left\langle M_{2} / M_{1}\right\rangle$ as a function of the impact velocity $v_{0}$ for all plate thicknesses $H$ considered. The main panel presents the same data rescaled with appropriate powers of the plate thickness $H$ to obtain collapse of the curves.

the system undergoes a transition as the impact velocity is varied from the damage phase $\left(v_{0}<v_{c}\right)$, characterized by the presence of a dominating piece, to the fragmentation phase $\left(v_{0}>v_{c}\right)$, where no major fragment prevails. The existence of the damage-fragmentation transition has been verified for various types of systems both by experiments [3-8,13] and computer simulations $[15,18,19,21,23-27]$.

In the inset of Fig. 3 the critical velocity $v_{c}$ is an increasing function of $H$ because the total mass of the sample $M_{\mathrm{tot}}$ increases with the plate thickness $M_{\text {tot }} \propto L^{2} H$. The main panel of Fig. 3 presents that rescaling $v_{0}$ and $\left\langle M_{2} / M_{1}\right\rangle$ of the inset with appropriate powers of the plate thickness $H$ the results obtained at different thicknesses can be collapsed on a master curve. The high quality collapse implies the validity of the scaling form

$$
\left\langle M_{2} / M_{1}\right\rangle\left(v_{0}, H\right)=H^{\beta} \phi\left(v_{0} / H^{\alpha}\right),
$$

where $\phi(x)$ denotes the scaling function. The exponents were obtained numerically as $\alpha=0.2$ (3) and $\beta=0.5(2)$ giving the best collapse in Fig. 3. It follows from Eq. (3) that the critical impact velocity $v_{c}$ increases as a power law of the plate thickness

$$
v_{c} \propto H^{\alpha}
$$

In the following we focus on the probability distribution of the mass of fragments $p(m)$ in the fragmented phase to understand how it evolves with the impact velocity at different plate thicknesses.

\section{MASS DISTRIBUTION OF FRAGMENTS}

Figure 4 presents the fragment mass distribution $p(m)$ for several values of the plate thickness $H$ at different impact velocities. A generic feature of the distributions is that in the damage phase $\left(v_{0}<v_{c}\right) p(m)$ is composed of two distinct parts: due to the presence of a big residue, a peak of the 

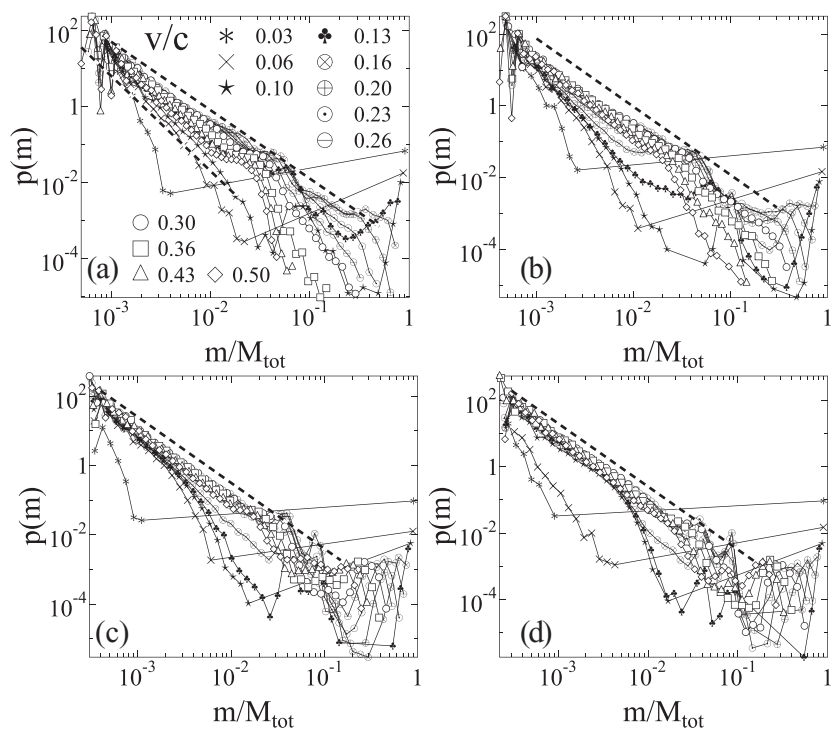

FIG. 4. Mass distribution of fragments at different impact velocities for four different plate thicknesses $H /\langle d\rangle$ : (a) 3, (b) 5, (c) 11, (d) 15. In the damage phase the distributions are composed of two distinct parts, i.e., the large residues form a peak at $m / M_{\text {tot }} \approx 1$, while the small fragments have a rapidly decreasing distribution. The two regimes are separated by a gap which gradually disappears as the critical velocity $v_{c}$ is approached from below. The dashed straight lines represent power laws of exponents (a) 1.7 and 2.4, (b) 1.7, (c) 1.9, and (d) 1.9 .

mass distribution is formed close to $m / M_{\text {tot }} \approx 1$ while the distribution of small pieces has a rapidly decreasing functional form. The two regimes are separated by a gap which gradually disappears as the critical impact velocity is approached from below. It can be observed that in the fragmented phase $\left(v_{0}>\right.$ $v_{c}$ ) small sized fragments have a power law mass distribution

$$
p(m) \propto m^{-\tau},
$$

which is followed by a cutoff regime. The power law over a broad range first occurs at the critical point.

The important feature of our results presented in Fig. 4 is that for low plate thicknesses $H$ we observe a gradual increase of the mass distribution exponent from $\tau=1.7$ obtained at the critical point $v_{c}$ to $\tau=2.4$ reached in the limit of high $v_{0}$ values [see Fig. 4(a)]. However, increasing the plate thickness in Figs. 4(b)-4(d) this dependence of $\tau$ on the impact velocity gradually disappears and for high plate thicknesses $H /\langle d\rangle \geqslant 11$ only a single value of the exponent $\tau=1.9$ remains. For increasing $v_{0}$ the mass of the largest fragment must decrease which may result in an apparent increase of the exponent simply due to the shifting cutoff of the distributions. However, contrary to Ref. [23] our analysis showed that the change of $\tau$ in Fig. 4 is the real behavior of $p(m)$; it cannot be transformed out by rescaling with the average fragment mass.

\section{A. Fragmentation mechanisms}

Our computer simulations revealed that the observed dependence of $\tau$ on the impact velocity is caused by a transition between two distinct fragmentation mechanisms, which emerges due to the interplay of the geometry of the

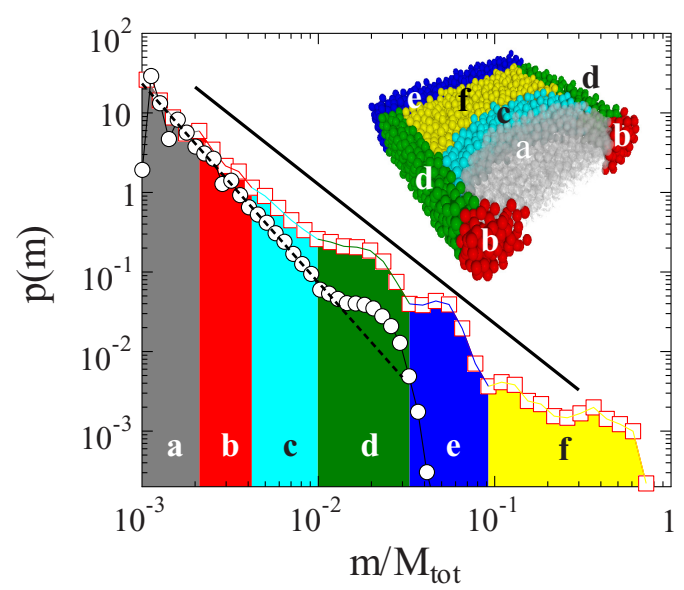

FIG. 5. (Color online) Mass distribution of fragments $p(m)$ obtained at the critical point of fragmentation $v_{c}$ for the smallest thickness $H /\langle d\rangle=3$. Fragments giving dominating contribution to different ranges of $p(m)$ originate from well defined spatial regions of the sample. These regions are highlighted in a single sample in the inset using the same colors (characters) as for the corresponding ranges of the mass distribution. For comparison we also present the mass distribution at the highest impact velocity $v_{0} / c=0.5$ where the power law regime is significantly steeper. The slopes of the two straight lines are $\tau=1.7$ and $\tau=2.4$.

sample and of the dimensionality of the embedding space. It can be seen in Fig. 2 that immediately after impact the specimen gets damaged in the vicinity of the impact site, i.e., in a small volume starting from the surface all beams get broken and single particles (powder in the model) are ejected from the specimen. The impact loading generates a shockwave which gets gradually attenuated by the breaking of beams and by the expansion over a larger volume. At sufficiently high $v_{0}$ the compression wave can pass through the sample and reflects back with opposite phase as a tensile wave at the free boundaries of the rectangular specimen freely evolving in the three-dimensional embedding space. In this velocity range the stress field which gives rise to the formation of extended cracks and final breakup is determined by the interference pattern of compression and reflected tensile waves. This mechanism has the consequence that for the limit of thin plates $H \ll L$ the breakup of the specimen at the critical impact velocity is caused by a relatively regular crack pattern which is essentially two-dimensional. To demonstrate how this fragmentation mechanism works Fig. 5 presents the mass distribution $p(m)$ of pieces at the critical impact velocity for $H /\langle d\rangle=3$ together with a sample in the inset where particles of different spatial regions are highlighted by different colors. It can be observed that $p(m)$ has a power law behavior over a broad range of fragment masses, however, it is decorated by distinct maxima. Detailed analysis revealed that the emergence of the maxima is the fingerprint of the regularity of the two-dimensional crack structure of the platelike object, i.e., fragments giving dominating contribution to a maximum always emerge in the same spatial region of the specimen. To make it clear in Fig. 5 we assigned colors (characters) to the maxima of $p(m)$ such that in the inset the same colors (characters) are used for the particles of the spatial regions 
where the corresponding fragments originate from. It can be seen that the smallest fragments comprising a few particles [grey (a)] are generated in the destructed zone close to the impact point, while larger fragments are formed deeper in the sample [cyan (c)]. The interference of elastic waves generates a highly stretched zone along the surface of the plate which gives rise to the detachment of surface fragments both on the left and right sides [green (d)] and on the back side [blue (e)]. The two corners of the front side of the sample [red (b)] result in a slight local maximum of $p(m)$ between the grey (a) and cyan (c) regions. The largest fragment [yellow (f)] controlling the cutoff of the distribution is created inside the specimen close to the back side with a shape elongated perpendicular to the direction of impact. The two maxima of the yellow region of $p(m)$ are caused by the fact that the yellow region breaks into two major pieces with a high probability.

As the impact velocity gets high enough the overall twodimensional character of the crack pattern disappears and most of the cracks are created in the three-dimensional bulk of the material. This second fragmentation mechanism gradually becomes dominating with increasing $v_{0}$. As a consequence, in Fig. 4(a) the cutoff of $p(m)$ shifts toward smaller $m$ and the fraction of large fragments decreases in the mass distribution giving rise to a higher value of the power law exponent $\tau$. It follows from the above arguments that the dependence of the mass distribution exponent $\tau$ on the impact velocity $v_{0}$ is caused by the gradual crossover from the planar two-dimensional to the three-dimensional bulk crack structure. At the critical velocity the $2 \mathrm{D}$ character dominates while in the limit of high $v_{0}$ the crack pattern is completely three-dimensional. In the intermediate velocity range both mechanisms are present so that the observed mass distribution is a blend of their contributions. The crossover is gradual in the sense that in the highly destroyed zone around the impact site the crack pattern is three-dimensional already at the lowest impact velocities which then spreads over the sample as $v_{0}$ increases. To have a clear view on the two limits of $p(m)$ with different exponents of the power law regimes in Fig. 5 the mass distribution is also presented for the highest impact velocity $v_{0} / c=0.5$ we considered. The two straight lines of the figure represent power laws of exponents $\tau=1.7$ and $\tau=2.4$.

\section{B. Superposition of subsets of fragments}

The changing exponent of the mass distribution is the consequence of the gradual crossover of the crack structure in the sample as the impact velocity increases. The two- and three-dimensional crack structures favor fragment formation in different spatial regions of the sample having also different extensions. Hence, in order to understand how the crossover emerges we identified sets of fragments according to their position in the sample and analyzed how their contributions to the complete mass distributions evolve with the impact velocity and plate thickness. The key feature of fragments is whether they are created in the bulk or on the surface of the initial body. Since the sample surface is rather irregular, to identify the position of fragments we construct their bounding box and compare the location of its corner points to the bounding box of the original sample. For this purpose in the final state of the fragmentation process we reassembled the sample, as it is illustrated in Fig. 2(d), and determined the bounding box of each fragment aligning the sides of the box with the edges of the sample. Based on the position and extension of the bounding box three types of fragments are distinguished:

Bulk fragment. If the corners of the bounding box all lie inside the sample, i.e., their distance from the surface of the bounding box of the sample is greater than a threshold distance $0.2\langle d\rangle$, the fragment is considered to be a bulk fragment.

Surface fragment. If any of the corners, but not all of them, are within the threshold distance to the surface of the sample's bounding box, the fragment is called surface fragment.

Spanning fragment. Those fragments which span the sample at least in one direction are called spanning fragments. For spanning fragments all the corners of the bounding box lie in the vicinity of the sample surface.

Figure 6 demonstrates the identification of the three subsets of fragments in two plates of different thicknesses. For each subset a single fragment is highlighted together with its bounding box.

The spanning fragments are typically formed by cracks, which connect two opposite sides of the sample. Such cracks emerge due to the global interference pattern of elastic waves. In thin plates below and at the critical impact velocity $v_{c}$ most

(a)

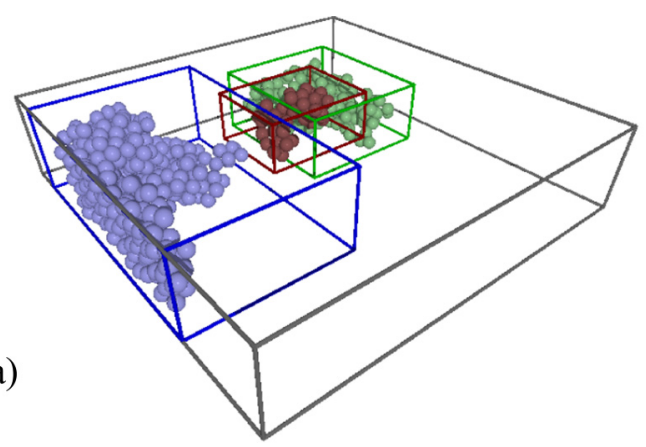

(b)

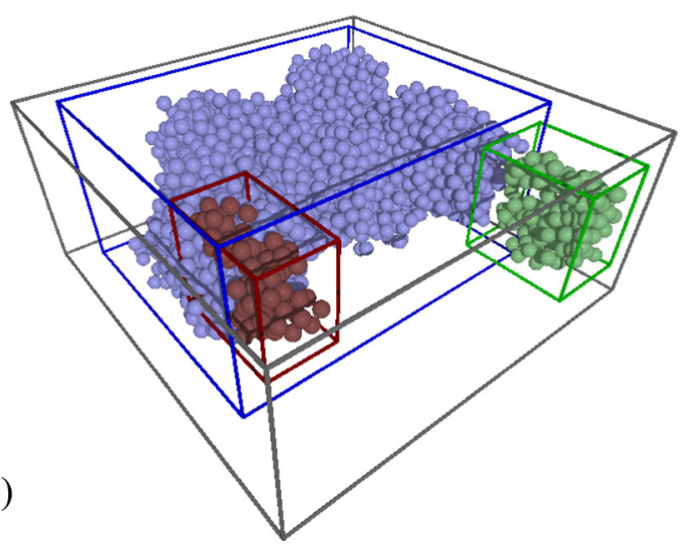

FIG. 6. (Color online) Identification of fragment subsets based on the bounding box of fragments and of the complete sample. Two plates are shown with different thicknesses and impact velocities (a) $H /\langle d\rangle=5$ and $v_{0} / c=0.23$, (b) $H /\langle d\rangle=11$ and $v_{0} / c=0.3$. For each subset the largest fragment is highlighted with different colors: light blue (light grey), green (medium grey), and red (dark grey) stand for the spanning, surface, and bulk fragments, respectively. The bounding boxes are indicated by the wire frames. 

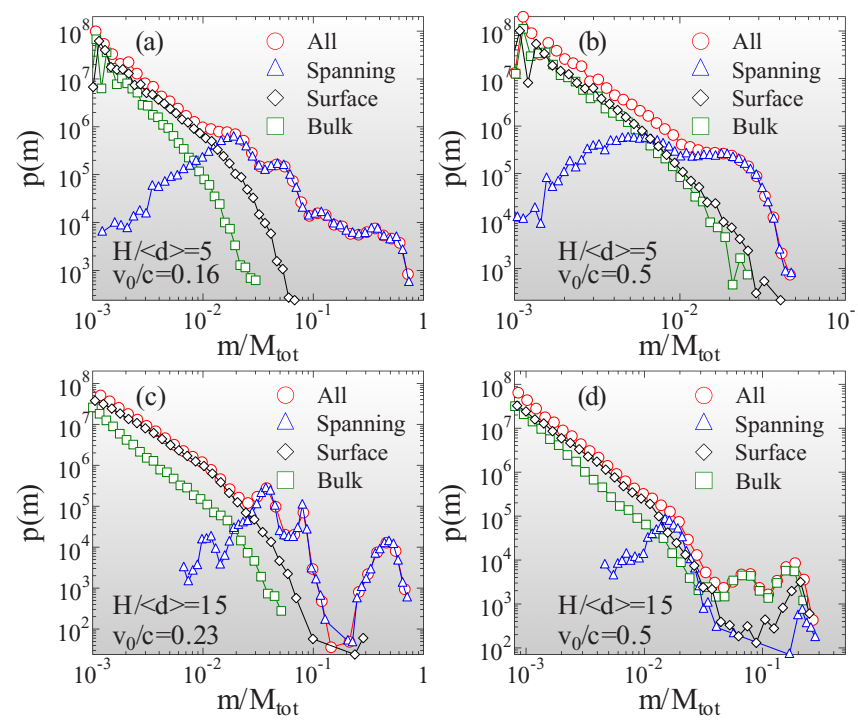

FIG. 7. (Color online) Mass distribution of subsets of surface, spanning, and bulk fragments together with the complete distribution of all fragments for two plate thicknesses $H /\langle d\rangle=5$ and 15 in the upper and lower rows, respectively. In both cases the results are presented for two values of the impact velocity $v_{0}$ slightly above the corresponding $v_{c}$ (left column) and for the limit of high speed impact (right column).

of the mass is comprised in spanning fragments; bulk and surface pieces can mainly be formed around the destroyed zone at the impact site. Above $v_{c}$ the fraction of spanning fragments rapidly decreases, however, they always have the largest mass so that the spanning fragments determine the cutoff of the mass distribution $p(m)$ at any $v_{0}$. Figure 7 presents the mass distribution of the three subsets of fragments for a plate of thickness $H /\langle d\rangle=5$ at the critical impact velocity together with the complete distribution. Note that the partial distributions are normalized such that their integral is equal to their fraction in the complete set of fragments. The figure clearly demonstrates that the cutoff and the large mass regime of $p(m)$ is controlled by fragments which span the sample in the direction perpendicular to the plate.

In thin plates surface fragments are generated from spanning fragments by cracks formed in the bulk of the specimen segmenting the spanning cracks. Due to this geometric constraint, in Fig. 7(a) the low mass regime of $p(m)$ is dominated by surface fragments, while bulk pieces have only a minor contribution. It follows that for thin plates the overall power law character of the complete mass distribution $p(m)$ originates from a mainly two-dimensional crack pattern. The power law regime of the complete mass distribution covers a broad range of fragment masses due to the spanning fragments and their daughter pieces on the surface of the sample. The exponent $\tau$ of the power law regime was obtained by fitting $\tau=1.7 \pm 0.05$ (compare also to Fig. 4).

Figure 7(b) presents the same mass distributions at the limit of high impact velocities. Here the range of the power law regime of the complete mass distribution $p(m)$ gets reduced and the exponent increases to a high value $\tau=2.4 \pm 0.07$ (compare also to Fig. 4). As the impact velocity increases bulk cracking gets activated which leads to a three-dimensional crack pattern with a highly disordered structure. Consequently, the difference of surface and bulk fragments disappears, they both have the same mass distribution with a high exponent in Fig. 7(b). Still the spanning fragments have the largest mass but they are only the remnants of the detached pieces.

Figures 7(c) and 7(d) present the corresponding results for 3D bulk bodies with $H /\langle d\rangle=15$. Compared to the platelike objects of Figs. 7(a) and 7(b) one can observe that the spanning pieces do not have a dominant role; at low velocities they are formed by detachment, while at high velocities they are just the corners of the sample. Spanning fragments give rise to distinct humps of the distribution at high $m$ values, however, also surface fragments have contribution to the cutoff of $p(m)$. Figure 4 demonstrated that for bulk bodies the fragment mass distribution exponent practically does not depend on the impact velocity, it has a unique value $\tau=1.9 \pm 0.05$. According to Figs. 7(c) and 7(d) the reason of the constant exponent is that the relative fraction of surface and bulk pieces does not depend on the impact velocity.

\section{Universality of partial mass distributions}

A very interesting outcome of our study is that in spite of the velocity dependence of the exponent observed for the complete mass distribution, the partial distributions of the subsets of bulk and surface fragments exhibit a high degree of universality. Figure 8 presents the scaling plot of the mass distributions of surface and bulk fragments obtained at different impact velocities for two plate thicknesses. It can be observed that rescaling the distributions with appropriate powers of the
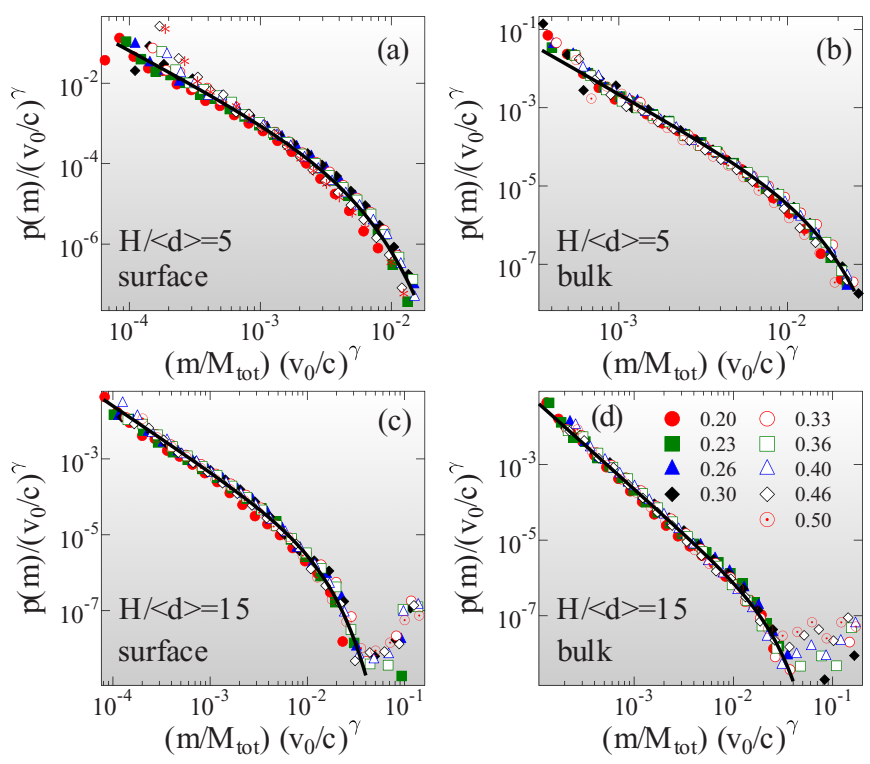

FIG. 8. (Color online) Data collapse analysis of the mass distribution of subsets of surface and bulk fragments for two plate thicknesses $H /\langle d\rangle=5$ and 15 in the upper and lower rows, respectively. Rescaling the distributions with appropriate powers of the impact velocity above the critical point good quality data collapse is achieved. The bold lines represent fits of the master curves with Eq. (7). 
impact velocity good quality data collapse is obtained in all cases. This scaling analysis demonstrates that the partial distributions obey the scaling law

$$
p(m)=v_{0}^{\gamma} \Psi\left(m v_{0}^{\gamma}\right)
$$

where the exponent $\gamma$ depends on the plate thickness $H$. Note that due to the normalization of the distributions the same exponent $\gamma$ has to be used along the horizontal and vertical axis. In Fig. 8 best collapse was obtained with the exponents $H /\langle d\rangle=5: \gamma=1.3$ (surface) and $\gamma=0.2$ (bulk); $H /\langle d\rangle=15: \gamma=0.7$ (surface) and $\gamma=0.25$ (bulk). The scaling function $\Psi(x)$ was fitted with the functional form

$$
\Psi(x) \propto x^{-\tau} \exp \left[-\left(x / x^{*}\right)^{\kappa}\right]
$$

where the exponent $\kappa$ and the characteristic scale $x^{*}$ only control the shape of the cutoff. The most remarkable feature of the results is that best fits of the scaling function $\Psi$ is obtained with $\tau=1.7$ and $\tau=2.4$ for surface and bulk fragments respectively, for all thicknesses. This result implies that the partial distributions exhibit universality as it has been observed for a broad class of fragmentation phenomena. The observed nonuniversality of the complete distribution of all fragments originates from the blending of the distributions of subsets of fragments whose contributions depend both on the impact velocity and on the plate thickness.

\section{DISCUSSION}

We investigated the impact induced breakup of heterogeneous brittle materials in the framework of a three-dimensional discrete element model focusing on the mass distribution of fragments. Based on large scale computer simulations we resolved recent debates on the universality of the power law exponent of the mass distribution which is crucial both from a scientific point of view and for industrial applications. Simulations were carried out to investigate the impact induced breakup of platelike objects where both the thickness of the plate and the impact velocity were varied in a broad range. Our computer simulations revealed that for thin plates embedded in the three-dimensional space the power law exponent of the fragment mass distribution has a strong dependence on the impact velocity: power law is first obtained at the critical velocity of impact with an exponent $\tau=1.7$ which then gradually increases to $\tau=2.4$ for high impact velocities. However, for $3 \mathrm{D}$ bulk samples a unique exponent is obtained, $\tau=1.9$; dependence on the impact velocity can only be pointed out for the cutoff of the distributions. Note that the value of the exponent $\tau=1.7$ of $p(m)$ falls close to the theoretical prediction of Refs. [41,42] based on the branching-merging scenario of dynamic cracks: if fragments are formed by the merging of branches of splitting unstable cracks a universal exponent of the fragment mass distribution $\tau=(2 D-1) / D$ was predicted depending solely on the dimensionality $D$ of the embedding space. For $D=3$ the formula yields $\tau=5 / 3$ in the vicinity of our numerical result, although, in our case simulations did not reveal a branching-merging sequence of cracks. The exponent $\tau=1.9$ of 3D bulk samples is consistent with other DEM results, e.g., the same exponent was obtained for the fragmentation of brittle spheres impacted against a hard wall $[21,23]$.

The reason of the velocity dependent exponent is that due to the interplay of the geometry of the sample and of the dimensionality of the embedding space a crossover occurs between two different fragmentation mechanisms. In the vicinity of the critical impact velocity the crack pattern is essentially two-dimensional determined by the interference pattern of compressive and tensile waves generated by the impact. This crack pattern has a high degree of regularity which gives rise to local maxima of the fragment mass distribution on the overall power law functional form. At increasing impact velocities bulk cracking gets activated so that the crack structure becomes three-dimensional with a high degree of randomness.

A similar effect of the interference pattern of elastic waves has been observed for slender rods where fragmentation was induced by a hit at the free rod end. The mass distribution of pieces proved to have discrete humps at certain fractions of the buckling wavelength [43] similar to what we obtained for plates. Studying the impact induced breakup of thin glass plates, in the experiments of Ref. [31] an increase of the mass distribution exponent was reported with increasing impact velocity. The authors argued that the effect can be attributed to the increase of the fractal dimension of the crack pattern, i.e., as the crack structure gets more and more space filling, the mass distribution exponent increases and approaches a limit value [31]. Our results clarify the background of these experimental findings unveiling the underlying mechanism.

Comparing the bounding box of fragments and that of the complete sample we decomposed the fragment ensemble into subsets of bulk, surface, and spanning pieces. The formation of these fragments is governed by different cracking mechanisms. Scaling analysis showed a striking universality of the mass distributions of bulk and surface fragments with strongly different exponents. The results imply that the velocity dependence of the exponent of the complete mass distribution at intermediate velocities $v_{0}$ is observed due to the mixing of the contributions of the subsets of fragments, where the mixing ratio depends on $v_{0}$. Our results have the general consequence that energy dependence of the mass distribution exponent of fragmentation phenomena can be expected when a low dimensional object is embedded into a higher dimensional space allowing for the emergence of a transition in the spatial structure of cracks generated by the initial shockwave.

\section{ACKNOWLEDGMENTS}

The work is supported by the Project No. TAMOP-4.2.2.A$11 / 1 /$ KONV-2012-0036. The project is implemented through the New Hungary Development Plan, co-financed by the European Union, European Social Fund an the European Regional Development Fund. We acknowledge the support of the project OTKA K84157. 
[1] J. A. Aström, Adv. Phys. 55, 247 (2006).

[2] W. J. Stronge, Impact Mechanics (Cambridge University Press, New York, 2000).

[3] Y. Hayakawa, Phys. Rev. B 53, 14828 (1996).

[4] H. Katsuragi, D. Sugino, and H. Honjo, Phys. Rev. E 68, 046105 (2003).

[5] H. Katsuragi, D. Sugino, and H. Honjo, Phys. Rev. E 70, 065103(R) (2004).

[6] H. Katsuragi, S. Ihara, and H. Honjo, Phys. Rev. Lett. 95, 095503 (2005).

[7] F. P. M. dos Santos, V. C. Barbosa, R. Donangelo, and S. R. Souza, Phys. Rev. E 81, 046108 (2010).

[8] W. Ji and A. M. Waas, Europhys. Lett. 89, 46003 (2010).

[9] D. L. Turcotte, J. Geophys. Res. 91, 1921 (1986).

[10] D. L. Turcotte, Fractals and Chaos in Geology and Geophysics (Cambridge University Press, Cambridge, UK, 1997).

[11] L. Oddershede, P. Dimon, and J. Bohr, Phys. Rev. Lett. 71, 3107 (1993).

[12] H. Inaoka, E. Toyosawa, and H. Takayasu, Phys. Rev. Lett. 78, 3455 (1997).

[13] J. A. Åström, R. P. Linna, J. Timonen, P. F. Møller, and L. Oddershede, Phys. Rev. E 70, 026104 (2004)

[14] F. K. Wittel, F. Kun, H. J. Herrmann, and B. H. Kröplin, Phys. Rev. Lett. 93, 035504 (2004).

[15] F. Kun, F. K. Wittel, H. J. Herrmann, B. H. Kröplin, and K. J. Maloy, Phys. Rev. Lett. 96, 025504 (2006).

[16] F. P. M. dos Santos, V. C. Barbosa, R. Donangelo, and S. R. Souza, Phys. Rev. E 84, 026115 (2011).

[17] J. P. Hooper, J. Appl. Phys. 112, 043508 (2012).

[18] F. Kun and H. J. Herrmann, Phys. Rev. E 59, 2623 (1999).

[19] B. Behera, F. Kun, S. McNamara, and H. J. Herrmann, J. Phys.: Condens. Matter 17, S2439 (2005).

[20] F. P. dos Santos, R. Donangelo, and S. Souza, Physica A 374, 680 (2007).

[21] H. A. Carmona, F. K. Wittel, F. Kun, and H. J. Herrmann, Phys. Rev. E 77, 051302 (2008).
[22] O. Durand and L. Soulard, J. Appl. Phys. 111, 044901 (2012).

[23] G. Timár, F. Kun, H. A. Carmona, and H. J. Herrmann, Phys. Rev. E 86, 016113 (2012).

[24] N. Myagkov and V. Stepanov, Physica A 410, 120 (2014).

[25] F. Kun and H. J. Herrmann, Comput. Methods Appl. Mech. Eng. 138, 3 (1996).

[26] F. K. Wittel, F. Kun, H. J. Herrmann, and B.-H. Kröplin, Phys. Rev. E 71, 016108 (2005).

[27] J. A. Åström, B. L. Holian, and J. Timonen, Phys. Rev. Lett. 84, 3061 (2000).

[28] G. Timár, J. Blömer, F. Kun, and H. J. Herrmann, Phys. Rev. Lett. 104, 095502 (2010).

[29] T. Ishii and M. Matsushita, J. Phys. Soc. Jpn. 61, 3474 (1992).

[30] E. S. Ching, S. Lui, and K.-Q. Xia, Physica A 287, 83 (2000).

[31] T. Kadono and M. Arakawa, Phys. Rev. E 65, 035107 (2002).

[32] T. Kadono, M. Arakawa, and N. K. Mitani, Phys. Rev. E 72, 045106(R) (2005).

[33] N. Myagkov and T. Shumikhin, Physica A 358, 423 (2005).

[34] N. Sator and H. Hietala, Int. J. Fract. 163, 101 (2010).

[35] N. Sator, S. Mechkov, and F. Sausset, Europhys. Lett. 81, 44002 (2008).

[36] F. Kun, I. Varga, S. Lennartz-Sassinek, and I. G. Main, Phys. Rev. E 88, 062207 (2013).

[37] F. Kun, I. Varga, S. Lennartz-Sassinek, and I. G. Main, Phys. Rev. Lett. 112, 065501 (2014).

[38] T. Pöschel and T. Schwager, Computational Granular Dynamics (Springer, Berlin, 2005).

[39] Computer Simulation of Liquids, edited by M. P. Allen and D. J. Tildesley (Oxford University Press, Oxford, 1984).

[40] T. Kadono, Phys. Rev. Lett. 78, 1444 (1997).

[41] J. A. Aström, F. Ouchterlony, R. P. Linna, and J. Timonen, Phys. Rev. Lett. 92, 245506 (2004).

[42] P. Kekalainen, J. A. Aström, and J. Timonen, Phys. Rev. E 76, 026112 (2007)

[43] J. R. Gladden, N. Z. Handzy, A. Belmonte, and E. Villermaux, Phys. Rev. Lett. 94, 035503 (2005). 